\title{
Variable frequency photonic crystals
}

\author{
Xiang-Yao $\mathrm{Wu}^{a *}$, Ji $\mathrm{Ma}^{a}$, Xiao-Jing $\mathrm{Liu}^{a}$ \\ Jing-Hai Yang ${ }^{a}$, Hong $\mathrm{Li}^{a}$ and Wan-Jin Chen ${ }^{a \dagger}$ \\ ${ }^{a}$ Institute of Physics, Jilin Normal University, Siping 136000 China
}

\begin{abstract}
In this paper, we have firstly proposed a new one-dimensional variable frequency photonic crystals (VFPCs), and calculated the transmissivity and the electronic field distribution of VFPCs with and without defect layer, and considered the effect of defect layer and variable frequency function on the transmissivity and the electronic field distribution. We have obtained some new characteristics for the VFPCs, which should be help to design a new type optical devices.
\end{abstract}

PACS: 41.20.Jb, 42.70.Qs, 78.20.Ci

Keywords: photonic crystals; variable frequency medium; transmissivity; electronic field distribution

PACS numbers:

\section{Introduction}

Photonic crystals are artificial materials with periodic variations in refractive index that are designed to affect the propagation of light, which were first introduced theoretically by Yablonovitch [1], and experimentally by John [2]. PCs, constructed with periodic structure of artificial dielectrics or metallic materials, have attracted many researchers in the past two decades for their unique electromagnetic properties and scientific and engineering applications [3-9]. These crystals indicate a range of forbidden frequencies, called photonic band gap, as a result of Bragg scattering of the electromagnetic waves passing through such a periodical structure $[10,11]$. As the periodicity of the structure is broken by introducing a layer with different optical properties, a localized defect mode will appear inside the band gap. Enormous potential applications of PCs with defect layers in different areas, such as light emitting diodes, filters and fabrication of lasers have made such structures are interesting research topic in this field.

Such materials are employed for the realization of diverse optical devices, as for example distributed feedback laser $[12,13]$ and optical switches $[14,15]$. The addition of defects in the periodic alternation, Or the realization of completely random sequences, results in disordered photonic structures [16-18]. In the case of one-dimensional disordered photonic structures, very interesting physical phenomena Have been theoretically predicted or experimentally observed.

In the paper, we have firstly proposed a new onedimensional variable frequency photonic crystals (VF$\mathrm{PCs}$ ), which is made up of variable frequency medium. The so-called variable frequency medium is a new type optical medium, which can change the light frequency when the light passes through the medium. For the conventional medium, it does not change light frequency and

\footnotetext{
*E-mail: wuxy2066@163.com

${ }^{\dagger}$ E-mail: chenwwjj@126.com
}

change the light wavelength. When light passes through a medium, it does not exchange energy with light, the light frequencies should not be changed, the medium is called conventional medium. When light passes through a medium, it exchange energy with light, the light frequencies should be changed, the medium is called variable frequency medium. We can make the photonic crystals with the variable frequency medium, which is called the variable frequency photonic crystals (VFPCs). We should studied the transmissivity and the electronic field distribution of the VFPCs and compare them with the conventional PCs (CPCs), and obtained some new new characteristics for the VFPCs, which should be help to design a new type optical devices.

\section{Transfer matrix, transmissivity and elec- tronic field distribution of PCs}

For one-dimensional conventional PCs, the calculations are performed using the transfer matrix method [19], which is the most effective technique to analyze the transmission properties of PCs. For the medium layer $i$, the transfer matrices $M_{i}$ for $T E$ wave is given by [19]:

$$
M_{i}=\left(\begin{array}{cc}
\cos \delta_{i} & -i \sin \delta_{i} / \eta_{i} \\
-i \eta_{i} \sin \delta_{i} & \cos \delta_{i}
\end{array}\right)
$$

where $\delta_{i}=\frac{\omega}{c} n_{i} d_{i} \cos \theta_{i}, c$ is speed of light in vacuum, $\theta_{i}$ is the ray angle inside the layer $i$ with refractive index $n_{i}=$ $\sqrt{\varepsilon_{i} \mu_{i}}, \eta_{i}=\sqrt{\varepsilon_{i} / \mu_{i}} \cos \theta_{i}, \cos \theta_{i}=\sqrt{1-\left(n_{0}^{2} \sin ^{2} \theta_{0} / n_{i}^{2}\right)}$, in which $n_{0}$ is the refractive index of the environment wherein the incidence wave tends to enter the structure, and $\theta_{0}$ is the incident angle.

The final transfer matrix $M$ for an $N$ period structure is given by:

$$
\begin{aligned}
\left(\begin{array}{c}
E_{1} \\
H_{1}
\end{array}\right) & =M_{B} M_{A} M_{B} M_{A} \cdots M_{B} M_{A}\left(\begin{array}{l}
E_{N+1} \\
H_{N+1}
\end{array}\right) \\
& =M\left(\begin{array}{l}
E_{N+1} \\
H_{N+1}
\end{array}\right)=\left(\begin{array}{ll}
A & B \\
C & D
\end{array}\right)\left(\begin{array}{l}
E_{N+1} \\
H_{N+1}
\end{array}\right),
\end{aligned}
$$




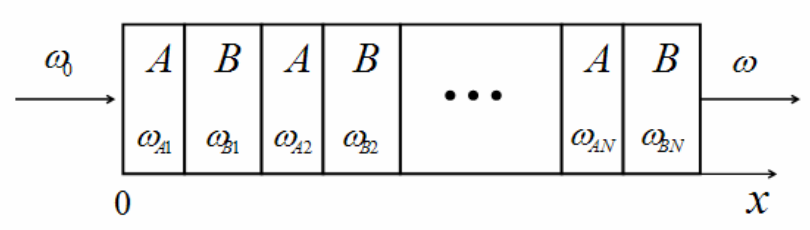

FIG. 1: one-dimensional variable frequency photonic crystals.

where

$$
M=\left(\begin{array}{cc}
A & B \\
C & D
\end{array}\right),
$$

with the total transfer matrix $M$, we can obtain the transmission coefficient $t$, and the transmissivity $T$, they are

$$
\begin{gathered}
t=\frac{E_{N+1}}{E_{1}}=\frac{2 \eta_{0}}{A \eta_{0}+B \eta_{0} \eta_{N+1}+C+D \eta_{N+1}}, \\
T=t \cdot t^{*}
\end{gathered}
$$

Where $\eta_{0}=\eta_{N+1}=\sqrt{\frac{\varepsilon_{0}}{\mu_{0}}}$.

The electronic field distribution at position $x$ is [19]

$$
\begin{aligned}
\left(\begin{array}{c}
E(x) \\
H(x)
\end{array}\right) & =M_{A}(a-x) M_{B}\left(M_{A} M_{B}\right)^{N-1}\left(\begin{array}{c}
E_{N+1} \\
H_{N+1}
\end{array}\right) \\
& =\left(\begin{array}{ll}
A^{\prime}(x) & B^{\prime}(x) \\
C^{\prime}(x) & D^{\prime}(x)
\end{array}\right)\left(\begin{array}{c}
E_{N+1} \\
H_{N+1}
\end{array}\right)
\end{aligned}
$$

with $E_{N+1}=E_{1} \cdot t$ and $H_{N+1}=\sqrt{\varepsilon_{0} / \mu_{0}} \cdot E_{N+1}$, we have

$$
E(x)=\left(A^{\prime}(x)+B^{\prime}(x) \sqrt{\varepsilon_{0} / \mu_{0}}\right) E_{1} \cdot t,
$$

and

$$
\left|\frac{E(x)}{E_{1}}\right|^{2}=\left|A^{\prime}(x)+B^{\prime}(x) \sqrt{\varepsilon_{0} / \mu_{0}}\right|^{2} \cdot|t|^{2} .
$$

\section{Transfer matrix and transmissivity of the VFPCs}

We have firstly proposed a new one-dimensional photonic crystals constituting by variable frequency medium. When light passes through the variable frequency medium, the light frequency can be written as

$$
\omega=f(n) \omega_{0},
$$

where $\omega_{0}$ is the incident light frequency, $\omega$ is the frequency of light in the variable frequency medium, $n$ is the refractive index of variable frequency medium, and $f(n)$ is called variable frequency function, which expresses the degree changing the light frequency for the variable frequency medium. When the variable frequency
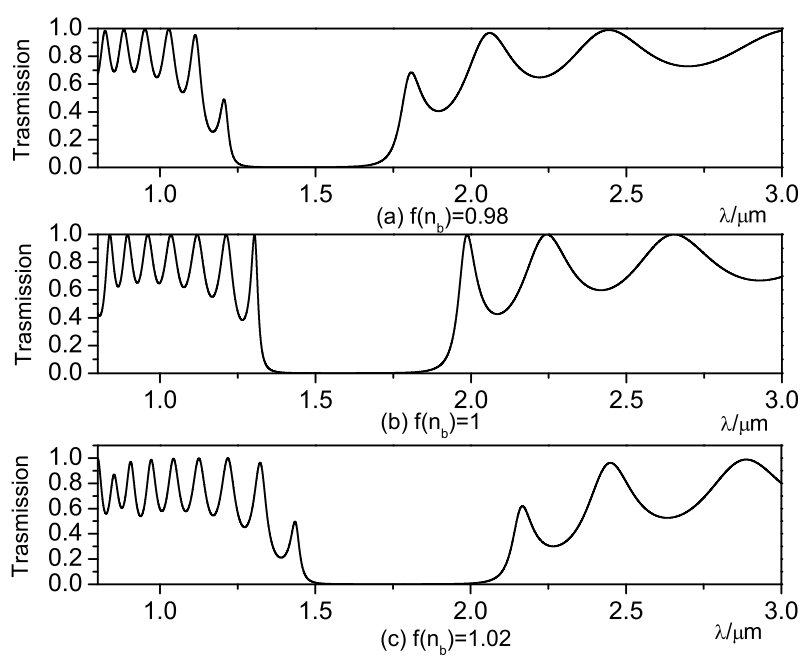

FIG. 2: Comparing the transmissivity of VFPCs with conventional PCs. (a) VFPCs $f\left(n_{b}\right)=0.98$, (b) conventional PCs $f\left(n_{b}\right)=1.0,(\mathrm{c}) \mathrm{VFPCs} f\left(n_{b}\right)=1.02$.
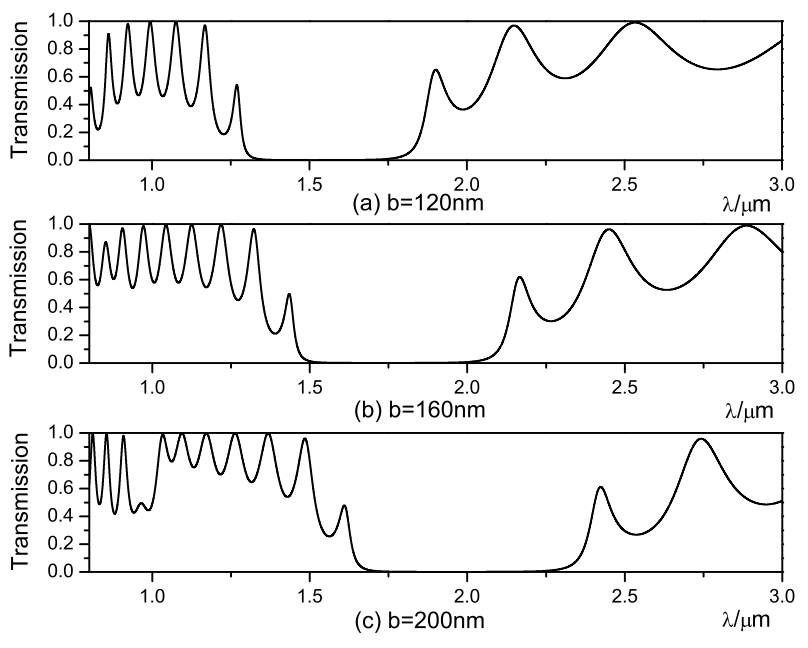

FIG. 3: The effect of the variable frequency medium thickness $b$ on the VFPCs transmissivity. (a) $b=120 \mathrm{~nm}$, (b) $b=$ $160 \mathrm{~nm}$, (c) $b=200 \mathrm{~nm}$.

function $f(n)>1$, the light frequency should be increased in variable frequency medium, when $f(n)<1$, the light frequency should be decreased. When $f\left(n_{b}\right)=1$ the variable frequency medium become the conventional medium.

For the VFPCs, its transfer matrices are similar to the conventional transfer matrices (1), they are

$\left.M_{A_{i}}=\left(\begin{array}{cc}\cos \delta_{a_{i}} & -i \sin \delta_{a_{i}} / \eta_{a} \\ -i \eta_{a} \sin \delta_{a_{i}} & \cos \delta_{a_{i}}\end{array}\right)(i=1,2, \cdots, N), 10\right)$ 
$M_{B_{i}}=\left(\begin{array}{cc}\cos \delta_{b_{i}} & -i \sin \delta_{b_{i}} / \eta_{b} \\ -i \eta_{b} \sin \delta_{b_{i}} & \cos \delta_{b_{i}}\end{array}\right)(i=1,2, \cdots, N)(11)$

For the structure of one-dimensional VFPCs $(A B)^{N}$, where the medium $B$ is the variable frequency medium, the medium $A$ is the conventional medium, and $N$ is the period numbers. The light frequencies of every medium are as follows:

$$
\begin{gathered}
\omega_{A 1}=\omega_{0}, \\
\omega_{B 1}=f\left(n_{b}\right) \omega_{0}, \\
\omega_{A 2}=\omega_{B 1}=f\left(n_{b}\right) \omega_{0}, \\
\omega_{B 2}=f\left(n_{b}\right) \omega_{A 2}=f^{2}\left(n_{b}\right) \omega_{0}, \\
\omega_{A i}=f^{i-1}\left(n_{b}\right) \omega_{0}, \\
\omega_{B i}=f^{i}\left(n_{b}\right) \omega_{0},
\end{gathered}
$$

which are shown in FIG. 1, and the corresponding phases are:

$$
\begin{gathered}
\delta_{a_{1}}=\frac{\omega_{0}}{c} \cdot n_{a} \cdot a, \\
\delta_{b_{1}}=\frac{f\left(n_{b}\right) \cdot \omega_{0}}{c} \cdot n_{b} \cdot b, \\
\delta_{a_{2}}=\frac{f\left(n_{b}\right) \cdot \omega_{0}}{c} \cdot n_{a} \cdot a, \\
\delta_{b_{2}}=\frac{f^{2}\left(n_{b}\right) \cdot \omega_{0}}{c} \cdot n_{b} \cdot b, \\
\delta_{a_{i}}=\frac{f^{i-1}\left(n_{b}\right) \cdot \omega_{0}}{c} \cdot n_{a} \cdot a, \\
\delta_{b_{i}}=\frac{f^{i}\left(n_{b}\right) \cdot \omega_{0}}{c} \cdot n_{b} \cdot b .
\end{gathered}
$$

\section{Numerical result}

In this section, we report our numerical results of VFPCs. The VFPCs main parameters are: The medium $A$ is conventional medium, its refractive indexes $n_{a}=1.38$, thickness $a=298 \mathrm{~nm}$, the medium $B$ is variable frequency medium, its refractive indexes $n_{b}=2.35$, thickness $b=160 \mathrm{~nm}$, and variable frequency function $f\left(n_{b}\right)$, the defect layer medium $D$ refractive indexes $n_{d}=2.97$, thickness $d=380 \mathrm{~nm}$. The structure of VFPCs is $(A B)^{8}$. In FIG. 2, we study the effect of the variable frequency
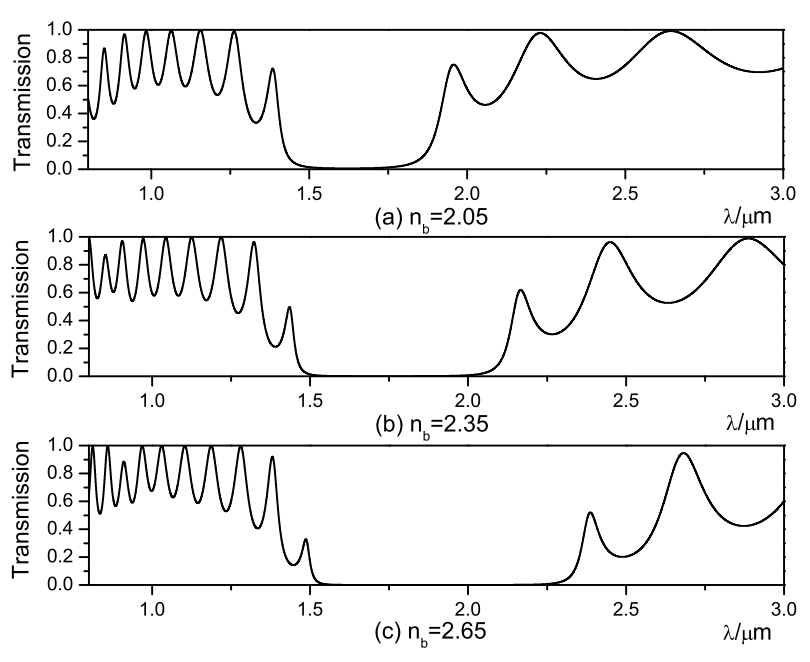

FIG. 4: The effect of the variable frequency medium refractive index $n_{b}$ on the VFPCs transmissivity. (a) $n_{b}=2.05$, (b) $n_{b}=2.35$, (c) $n_{b}=2.65$.
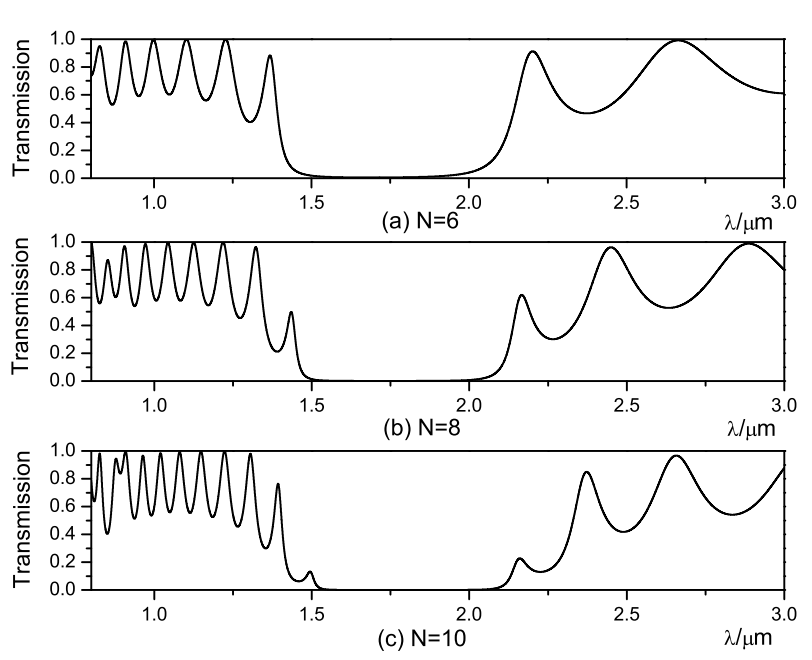

FIG. 5: The effect of the period number $N$ on the VFPCs transmissivity. (a) $N=6$, (b) $N=8$, (c) $N=10$.

function $f\left(n_{b}\right)$ on VFPCs transmissivity, the variable frequency function $f\left(n_{b}\right)$ in the FIG. $2(\mathrm{a})$, (b) and (c) are $0.98,1$ and 1.02, respectively. The FIG. 2 (b) is the transmissivity of conventional PCs because of $f\left(n_{b}\right)=1$, and FIG. 2 (a) and (c) are the transmissivity of VFPCs because of $f \neq 1$. Comparing the transmissivity of VFPCs with conventional PCs, we can obtain the new results: (1) When the variable frequency function $f\left(n_{b}\right)<1$ (FIG. $\left.2(\mathrm{a})\right)$, the band gaps blue shift, and the band gaps width decrease. (2) When the variable frequency function $f\left(n_{b}\right)>1$ (FIG. $2(\mathrm{c})$ ), the band gaps red shift, and the band gaps width increase. From the re- 

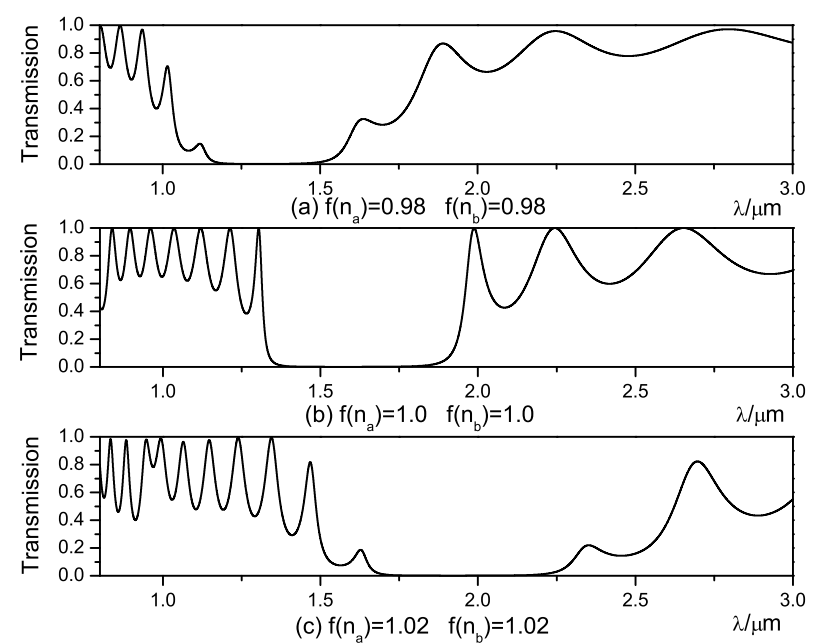

FIG. 6: The effect of the variable frequency functions $f\left(n_{a}\right)$ and $f\left(n_{b}\right)$ on the VFPCs transmissivity. (a) $f\left(n_{a}\right)=0.98$ $f\left(n_{b}\right)=0.98$, (b) $f\left(n_{a}\right)=1.0 f\left(n_{b}\right)=1.0$, (c) $f\left(n_{a}\right)=1.02$ $f\left(n_{b}\right)=1.02$.
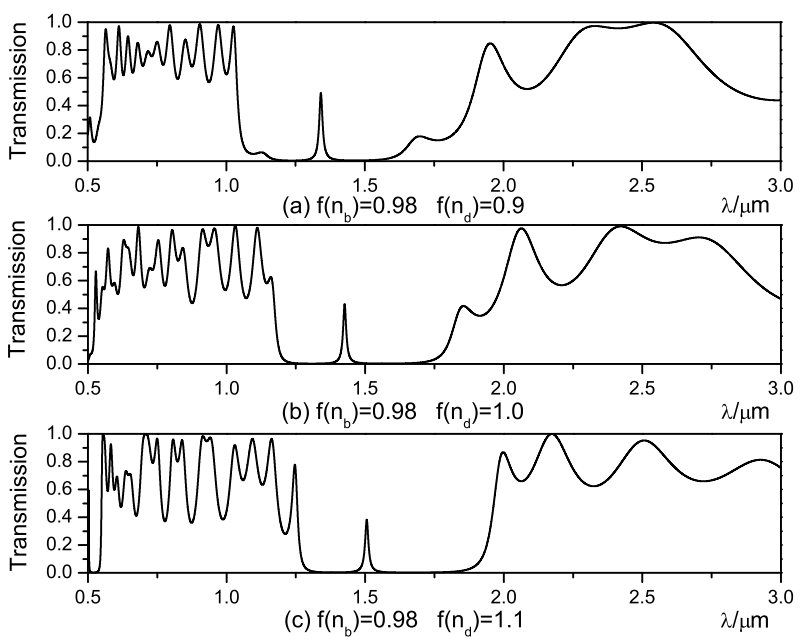

FIG. 7: The effect of the defect layer variable frequency function $f\left(n_{d}\right)$ on the structure $(A B)^{4} D(A B)^{4}$ VFPCs transmissivity. (a) $f\left(n_{b}\right)=0.98, f\left(n_{d}\right)=0.9$, (b) $f\left(n_{b}\right)=0.98$, $f\left(n_{d}\right)=1.0$, (c) $f\left(n_{b}\right)=0.98, f\left(n_{d}\right)=1.1$.

sults (1) and (2), we find the variable frequency function is an important factor of effect on transmissivity.

In FIG. 3, we study the effect of the variable frequency medium thickness on the VFPCs transmissivity, wherein variable frequency function $f\left(n_{b}\right)=1.02$. The FIG. 3 (a), (b) and (c) thickness $b$ are $120 \mathrm{~nm}, 160 \mathrm{~nm}$ and $200 \mathrm{~nm}$, respectively. From FIG. 3 (a), (b) and (c), we can find when the variable frequency medium thickness b increase the band gaps red shift, and the band gaps width in-
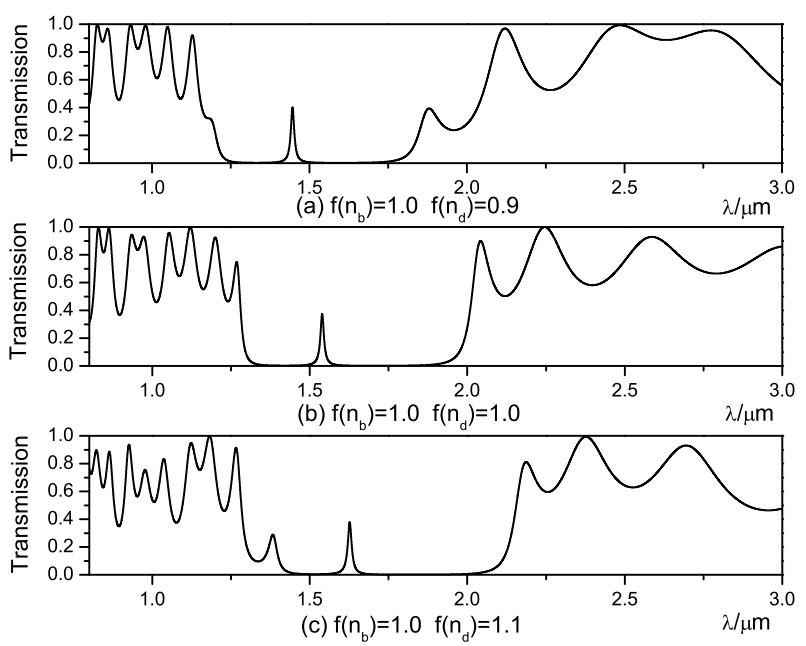

FIG. 8: The effect of the defect layer variable frequency function $f\left(n_{d}\right)$ on the structure $(A B)^{4} D(A B)^{4}$ VFPCs transmissivity. (a) $f\left(n_{b}\right)=1.0, f\left(n_{d}\right)=0.9$, (b) $f\left(n_{b}\right)=1.0$, $f\left(n_{d}\right)=1.0,(\mathrm{c}) f\left(n_{b}\right)=1.0, f\left(n_{d}\right)=1.1$.
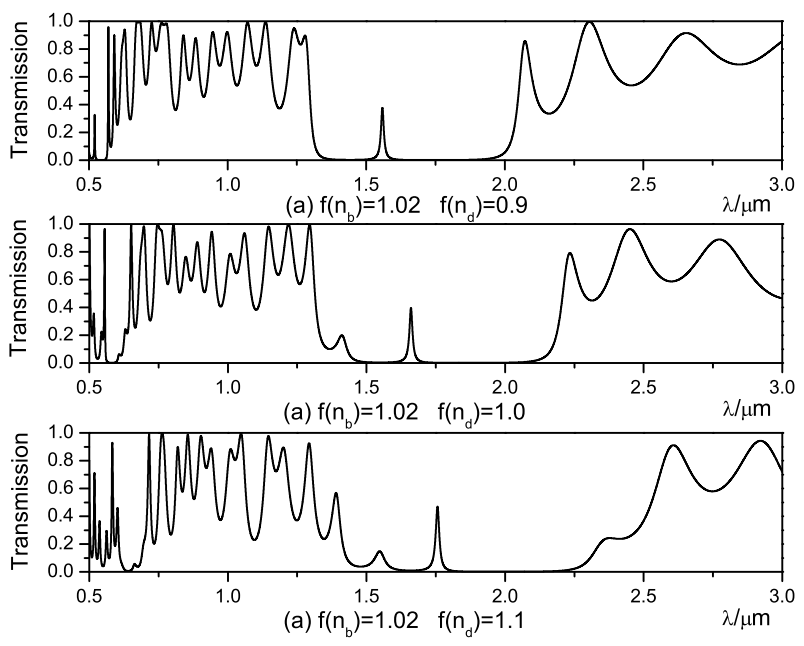

FIG. 9: The effect of the defect layer variable frequency function $f\left(n_{d}\right)$ on the structure $(A B)^{4} D(A B)^{4}$ VFPCs transmissivity. (a) $f\left(n_{b}\right)=1.02, f\left(n_{d}\right)=0.9$, (b) $f\left(n_{b}\right)=1.02$, $f\left(n_{d}\right)=1.0,\left(\right.$ c) $f\left(n_{b}\right)=1.02, f\left(n_{d}\right)=1.1$.

crease. In FIG. 4, we study the effect of the variable frequency medium refractive index on the VFPCs transmissivity, wherein variable frequency function $f\left(n_{b}\right)=1.02$. The FIG. 4 (a), (b) and (c) refractive index $n_{b}$ are 2.05, 2.35 and 2.65, respectively. From FIG. 4 (a), (b) and (c), we can find when the variable frequency medium refractive index $n_{b}$ increase the band gaps red shift, and the band gaps width increase. In FIG. 5, we study the effect of the period number $N$ on the VFPCs transmissivity, 
wherein variable frequency function $f\left(n_{b}\right)=1.02$. The FIG. 5 (a), (b) and (c) period number $N$ are 6, 8 and 10, respectively. From FIG. 5 (a), (b) and (c), we can find when the VFPCs period numbers increase the band gaps width increase. In the conventional PCs, the band gaps width are unchanged when the period numbers change. In FIG. 6, we consider the media $A$ and $B$ are all variable frequency medium, and study the effect of the variable frequency functions $f\left(n_{a}\right)$ and $f\left(n_{b}\right)$ on VFPCs transmissivity, the variable frequency functions $f\left(n_{a}\right)$ and $f\left(n_{b}\right)$ in the FIG. 6 (a), (b) and (c) are all 0.98, 1 and 1.02, respectively. Comparing the transmissivity of VFPCs with conventional PCs (FIG. 6 (b)), we can obtain the new results: (1) When the variable frequency functions $f\left(n_{a}\right)<1$ and $f\left(n_{b}\right)<1$ (FIG. 6 (a)), the band gaps blue shift, and the band gaps width decrease obviously. (2) When the variable frequency functions $f\left(n_{a}\right)>1$ and $f\left(n_{b}\right)>1$ (FIG. $6(\mathrm{c})$ ), the band gaps red shift, and the band gaps width increase obviously. In FIGs. 7, 8 and 9, we should study the effect of defect layer on the transmissivity, the structures of VFPCs are $(A B)^{4} D(A B)^{4}$, the variable frequency function of medium $B f\left(n_{b}\right)=0.98$, $f\left(n_{b}\right)=1.0$ and $f\left(n_{b}\right)=1.02$ corresponding to FIGs. 7, 8 and 9 , the medium $A$ is conventional medium. For the defect layer medium $D$, its thickness, refractive index are: $d=380 \mathrm{~nm}$ and $n_{d}=2.97$, and the variable frequency function $f\left(n_{d}\right)$ are $0.9,1.0$ and 1.1 corresponding to the figures (a), (b) and (c) of FIGs. 7 to 9 . From FIGs. 7 to 9 , we can find when the variable frequency function of defect layer $f n_{d}$ increases, the defect model position red shift and intensity decreases. In the FIG. 10, the VFPCs structure is $(A B)^{4} D(A B)^{4}$, the variable frequency functions of medium $B$ and defect layer medium $D$ are $f\left(n_{b}\right)=0.98$ and $f\left(n_{d}\right)=1.1$, and the medium $A$ is conventional medium. In the FIG. 10 (a), (b) and (c), the refractive indices of defect layer medium $D$ are $n_{d}=2.97+i 0.02$ (absorbing medium), $n_{d}=2.97$ (conventional medium) and $n_{d}=2.97-i 0.02$ (active medium), respectively. Comparing with the conventional medium defect layer (FIG. 10 (b)), we can find the absorbing medium defect layer (FIG. 10 (a)) make the defect model intensity decrease and the active medium defect layer (FIG. 10 (c)) make the defect model intensity increase. In FIG. 11, we calculate the electronic field distribution of the VFPCs structure $(A B)^{8}$, and consider the influence of variable frequency function $f\left(n_{b}\right)$ on VFPCs electronic field distribution. The variable frequency function $f\left(n_{b}\right)=0.97,1$ and 1.03 are corresponding to the dash dot line, solid line and dot line of electronic field distribution, respectively. comparing with the conventional PCs $\left(f\left(n_{b}\right)=1\right)$, we can obtain new results: (1) When the variable frequency function $f\left(n_{b}\right)<1$ the peak values of VFPCs electronic field distribution decreased, the distribution curve left shift. (2) When the variable frequency function $f\left(n_{b}\right)>1$ the peak values of VFPCs electronic field distribution increased, the distribution curve right shift. In FIGs. 12 and 13, the VFPCs structure is $(A B)^{4} D(A B)^{4}$, the medium $A$ and defect
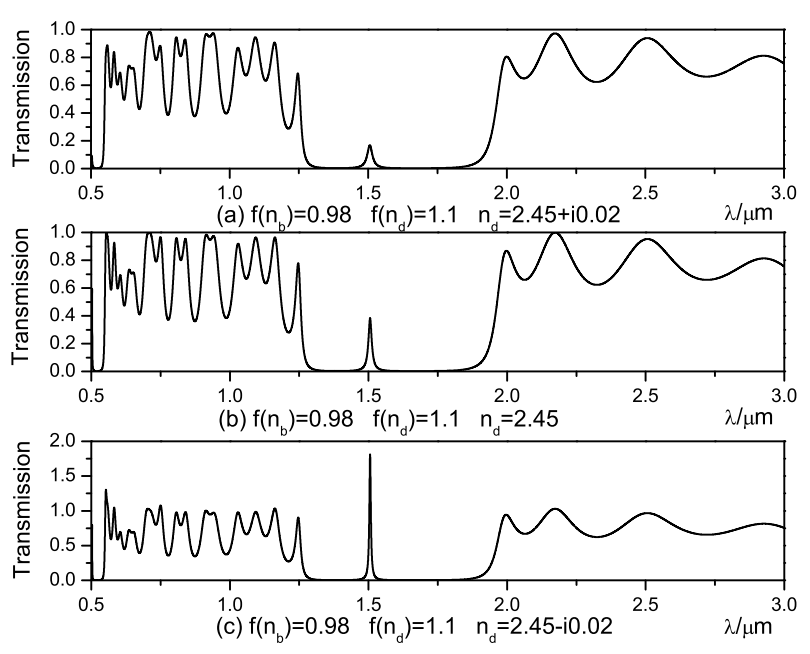

FIG. 10: The effect of the defect layer refractive index on the structure $(A B)^{4} D(A B)^{4}$ VFPCs transmissivity. (a) $n_{d}=$ $2.59+i 0.02$, (b) $n_{d}=2.59$, (c) $n_{d}=2.59-i 0.02$.

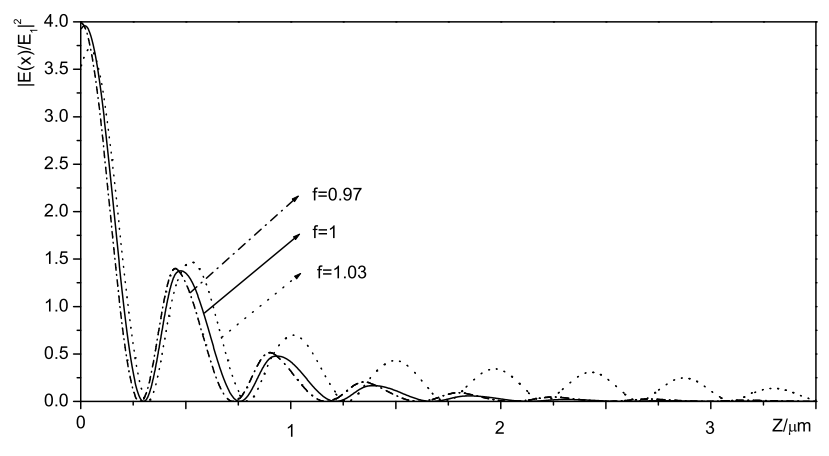

FIG. 11: The effect of the variable frequency functions $f\left(n_{b}\right)$ on the electronic field distribution $\left|E(x) / E_{1}\right|^{2}$ for the structure $(A B)^{8}$ VFPCs. (a) $f\left(n_{b}\right)=0.97$ dash dot line, (b) $f\left(n_{b}\right)=1$ solid line, (c) $f\left(n_{b}\right)=1.03$ dot line.

layer medium $D$ are conventional media and medium $B$ is the variable frequency medium. We consider the effect of the defect layer on electronic field distribution of VFPCs. In FIG. 12, the variable frequency function of medium $B f\left(n_{b}\right)=0.98$ and the defect layer medium $D$ refractive index $n_{d}=2.59$, thickness $d=200 \mathrm{~nm}$. In FIG. 13, the variable frequency function of medium $B$ $f\left(n_{b}\right)=1.02$ and the defect layer medium $D$ refractive index $n_{d}=2.59$, thickness $d=88 \mathrm{~nm}$. Comparing FIGs. 12 and 13 with FIG. 11, we can find the electronic field distribution has been local enhanced in the vicinity of defect layer.

\section{Conclusion}

In summary, we have studied the transmissivity and 


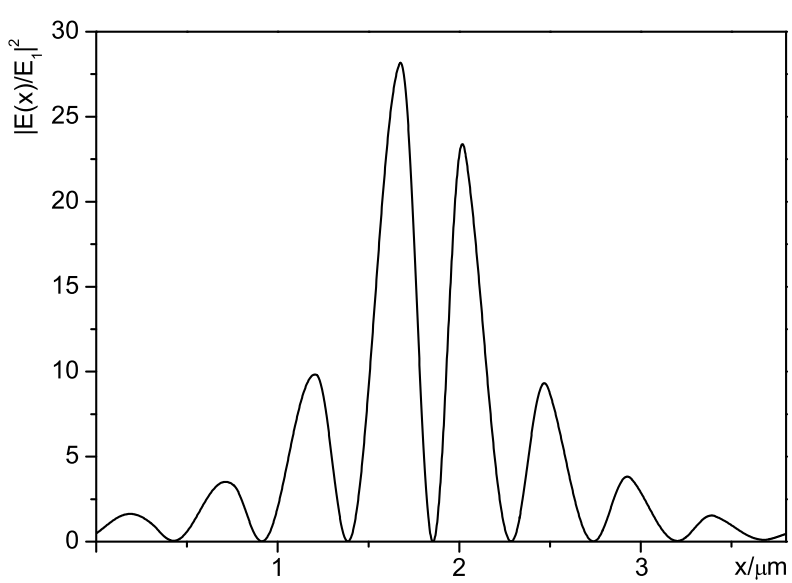

FIG. 12: The effect of the defect layer $D$ on the electronic field distribution $\left|E(x) / E_{1}\right|^{2}$ for the structure $(A B)^{4} D(A B)^{4}$ VFPCs. The $f\left(n_{b}\right)=0.98$ and $d=200 \mathrm{~nm}$.

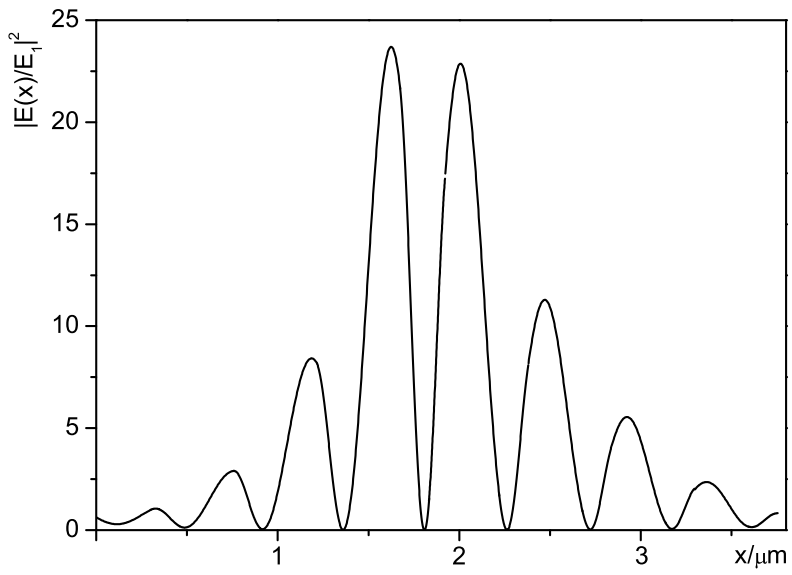

FIG. 13: The effect of the defect layer $D$ on the electronic field distribution $\left|E(x) / E_{1}\right|^{2}$ for the structure $(A B)^{4} D(A B)^{4}$ VFPCs. The $f\left(n_{b}\right)=1.02$ and $d=88 \mathrm{~nm}$.

the electronic field distribution of one-dimensional VFPCs with and without defect layer, and compare them with the conventional PCs. We obtained some new results: (1) When the variable frequency function $f\left(n_{b}\right)<$ 1 , the band gaps blue shift, and the band gaps width decrease. (2) When the variable frequency function $f\left(n_{b}\right)>1$, the band gaps red shift, and the band gaps width increase, i.e., the variable frequency function is an important factor of effect on transmissivity. (3) When the VFPCs period numbers increase the band gaps width increase. In the conventional PCs, the band gaps are unchanged when the period numbers increase. (4) When there is defect layer, we can find when the variable fre- quency function of defect layer $f n_{d}$ increases, the position of defect model red shift and its intensity decreases. (5) Comparing with the conventional medium, we can find the absorbing medium make the defect model intensity decrease and the active medium make the defect model intensity increase. (6) When the variable frequency function $f\left(n_{b}\right)<1$ the peak values of VFPCs electronic field distribution decreased, the distribution curve left shift. (7) When the variable frequency function $f\left(n_{b}\right)>1$ the peak values of VFPCs electronic field distribution increased, the distribution curve right shift. (8) When there is defect layer, we can find the electronic field distribution has been local enhanced in the vicinity of defect layer. These new characteristics of VFPCs should be help to design a new type optical devices.

\section{Acknowledgment}

This work is supported by Scientific and Technological Development Foundation of Jilin Province, Grant Number: 20130101031JC. 
[1] Yablonovitch, E., Phys. Rev. Lett., Vol. 58, 2059-2062, 1987.

[2] John, S., Phys. Rev. Lett., Vol. 58, 2486-2489, 1987.

[3] Noda, S., A. Chutinan, and M. Imada, Nature, Vol. 407, 608-610, 2000.

[4] Massaoudi, S., A. de Lustrac, and I. Huynen, J. Electromagn. Waves Appl., Vol. 14, 1967-1980, 2006.

[5] M. Gu, B. Jia, J. Li, M. Ventura, Laser Photonics Rev. 4, 414, 2010.

[6] T. Ergin, N. Stenger, P. Brenner, J. B. Pendry, M. Wegener, Science 328, 337, 2010.

[7] M. D. Turner, M. Saba, Q. Zhang, B. P. Cumming, G. E. Schroer-Turk, M. Gu, Nat. Photonics 7, 801, 2013.

[8] Mandal, B. and A. R. Chowdhury, J. Electromagn. Waves Appl., Vol. 1, 123-135, 2007.

[9] Zheng, Q. R., B. Q. Lin, and N. C. Yuan, J. Electromagn. Waves Appl., Vol. 2, 199-213, 2007.

[10] Yablonovitch, E., Phys. Rev. Lett., Vol. 67, 2295-2298, 1991.

[11] Joannopoulos, J. D., S. G. Johnson, J. N. Winn, and R.
D. Meada, Princeton University Press, Princeton, 2008.

[12] H. G. Park, S. H. Kim, S. H. Kwon, Y. G. Ju, J. K. Yang, J. H. Baek, Et al., Science. 305 (2004)

[13] F. Scotognella, A. Monguzzi, M. Cucini, F. Meinardi, D. Comoretto, R. Tubino, Int. J. Photoenergy. 2008 (2008) $1-4$.

[14] L. Criante, F. Scotognella, J. Phys. Chem. C. 116 (2012)21572-21576.

[15] Z. Hu, H. Wu, S. Gao, T. Wang, Opt. Commun. 288 (2013) 76-81.

[16] D.S. Wiersma, Nat. Photonics. 7 (2013) 188-196.

[17] M. Segev, Y. Silberberg, D.N. Christodoulides, Nat. Photonics. 7 (2013)197-204.

[18] P.A. Kalozoumis, C. Morfonios, N. Palaiodimopoulos, F.K. Diakonos, P. Schmelcher, Phys. Rev. A. 88 (2013) 033857.

[19] J. Zi, J. wan and C. Zhang, Appl. Phys. Lett, 73, 2084 (1998) 